\title{
Establishing core outcome domains in pediatric kidney disease: report of the Standardized Outcomes in Nephrology - Children and Adolescents (SONG-KIDS) consensus workshops
}

\section{Authors first and last names and highest degree:}

Camilla S. Hanson, $\mathrm{PhD}^{1,2}$, Jonathan C. Craig, $\mathrm{PhD}^{3}$, Charlotte Logeman, $\mathrm{MPH}^{1,2}$, Aditi Sinha, $\mathrm{MD}^{4}$, Allison Dart, $\mathrm{MD}^{5}$, Allison A. Eddy, $\mathrm{MD}^{6}$, Chandana Guha, MA ${ }^{1,2}$, Debbie S. Gipson, $\mathrm{MD}^{7}$, Detlef Bockenhauer, $\mathrm{PhD}^{8}$, Hui-Kim Yap, $\mathrm{MD}^{9}$, Jaap Groothoff, $\mathrm{PhD}^{10}$, Michael Zappitelli, $\mathrm{MD}^{11}$, Nicholas J.A Webb, MD ${ }^{12}$, Stephen I. Alexander, $\mathrm{MD}^{2}$, Susan L. Furth, $\mathrm{PhD}^{13}$, Susan Samuel, $\mathrm{MD}^{14}$, Alicia Neu, $\mathrm{MD}^{15}$, Andrea K. Viecelli, $\mathrm{PhD}^{16,17}$, Angela Ju, $\mathrm{PhD}^{1,2}$, Ankit Sharma, $\mathrm{MBBS}^{1,2}$, Eric H. Au, MBBS ${ }^{1,2}$, Hailey Desmond, MS ${ }^{7}$, Jenny I. Shen, MD ${ }^{18}$, Karine E. Manera, MIPH ${ }^{1,2}$, Karolis Azukaitis, MD ${ }^{19}$, Louese Dunn, $\mathrm{MPH}^{20}$, Simon A. Carter, MBBS ${ }^{2,21}$, Talia Gutman, $\mathrm{MPH}^{1,2}$, Yeoungjee Cho, $\mathrm{PhD}^{16,17}$, Amanda Walker, $\mathrm{MD}^{21,22}$, Anna Francis, $\mathrm{PhD}^{23}$, Cheryl Sanchez-Kazi, $\mathrm{MD}^{24}$, Joshua Kausman, $\mathrm{PhD}^{21}$, Meghan Pearl, $\mathrm{MD}^{25}$, Nadine Benador, $\mathrm{MD}^{26}$, Shobha Sahney, $\mathrm{MD}^{25}$, Allison Tong, $\mathrm{PhD}^{1,2}$

\section{Affiliations:}

${ }^{1}$ Sydney School of Public Health, The University of Sydney, Sydney, Australia

${ }^{2}$ Centre for Kidney Research, The Children's Hospital at Westmead, Sydney, Australia

${ }^{3}$ College of Medicine and Public Health, Flinders University, Australia

${ }^{4}$ Division of Nephrology, Department of Pediatrics, All India Institute of Medical Sciences, New Delhi, India.

${ }^{5}$ Department of Pediatrics and Child Health, The Children's Hospital Research Institute of Manitoba, University of Manitoba, Winnipeg, MB, Canada.

${ }^{6}$ Department of Pediatrics, University of British Columbia, Vancouver, BC, Canada.

${ }^{7}$ Division of Nephrology, Department of Pediatrics, University of Michigan, Ann Arbor, Michigan, USA 
${ }^{8}$ UCL Department of Renal Medicine Great Ormond Street Hospital for Children NHS Foundation Trust, London, UK.

${ }^{9}$ Department of Pediatrics, Yong Loo Lin School of Medicine, National University of Singapore, Singapore, Singapore.

${ }^{10}$ Department of Pediatric Nephrology, Emma Children's Hospital AMC Academic Medical Center, Amsterdam, The Netherlands.

${ }^{11}$ Department of Nephrology, SickKids, Toronto, Canada.

${ }^{12}$ Department of Paediatric Nephrology and NIHR/Wellcome Trust Clinical Research Facility University of Manchester, Manchester Academic Health Science Centre, Royal Manchester Children's Hospital Manchester, UK

${ }^{13}$ Department of Pediatrics, Perelman School of Medicine at the University of Pennsylvania, Children's Hospital of Philadelphia, Philadelphia, Pennsylvania, USA

${ }^{14}$ Department of Pediatrics, Section of Nephrology, University of Calgary, Calgary, AB, Canada.

${ }^{15}$ Division of Pediatric Nephrology, The Johns Hopkins University School of Medicine, Baltimore, USA

${ }^{16}$ Department of Nephrology, Princess Alexandra Hospital, Brisbane, Australia;

${ }^{17}$ Australasian Kidney Trials Network, The University of Queensland, Brisbane, Australia;

${ }^{18}$ Division of Nephrology and Hypertension, Lundquist Institute at Harbor-UCLA Medical Center, Torrance, USA

${ }^{19}$ Center of Pediatrics, Institute of Clinical Medicine, Faculty of Medicine, Vilnius University, Vilnius, Lithuania

${ }^{20}$ Sheffield Teaching Hospitals NHS Foundation Trust, S5 7AU, Sheffield, UK

${ }^{21}$ Department of Nephrology and Murdoch Children's Research Institute, Royal Children's Hospital, Melbourne, Victoria, Australia

${ }^{22}$ Department of Pediatrics, University of Melbourne, Victoria, Australia

${ }^{23}$ Child and Adolescent Renal Service, Queensland Children's Hospital, Brisbane, Queensland, Australia 
${ }^{24}$ Department of Pediatrics, Loma Linda University Children's Hospital, Loma Linda, California, United

States

${ }^{25}$ Department of Pediatrics, Division of Nephrology, University of California Los Angeles, David Geffen School of Medicine at UCLA, Los Angeles, USA

${ }^{26}$ Rady Children's Hospital, University of California at San Diego, San Diego, USA

\section{Corresponding author:}

Camilla Sara Hanson

Centre for Kidney Research, The Children's Hospital at Westmead, Westmead, NSW 2145, Sydney, Australia

Phone: +61 298451466 Fax: +61 298451491 Email: camilla.hanson@ sydney.edu.au

\section{Sources of support}

CSH is supported by the NHMRC Program Grant (ID1092957). AT is supported by a NHMRC Fellowship (ID1106716). SAC is supported by the NHMRC Post-graduate Scholarship (ID 1168994). AKV is supported by a Jacquot Research Establishment Fellowship and a Princess Alexandra Research Foundation Grant. EHA is supported by a NHMRC Postgraduate Scholarship (ID 1168606) and a RACP Jacquot NHMRC Award for Excellence. TG is supported by a NHMRC Postgraduate Scholarship (ID1 169149). The study was funded by The University of Sydney Research Accelerator Grant and the NHMRC Program Grant Better Evidence and Translation in Chronic Kidney Disease (BEAT-CKD) (ID1092957). The authors have no conflicts of interest relevant to this article to disclose.

Running headline: Core outcomes in children with CKD 


\begin{abstract}
Trials in children with chronic kidney disease (CKD) do not consistently report outcomes that are critically important to patients and caregivers. This can diminish the relevance and reliability of evidence for decisionmaking, limiting the implementation of results into practice and policy. As part of the Standardized Outcomes in Nephrology - Children and Adolescents (SONG-Kids) initiative, we convened two consensus workshops in San Diego, United States (7 patients, 24 caregivers, 43 health professionals; and Melbourne, Australia (7 patients, 23 caregivers, 49 health professionals). This report summarizes the discussions on the identification and implementation of the SONG-Kids core outcomes set. Four themes were identified; survival and life participation are common high priority goals, capturing the whole child and family, ensuring broad relevance across the patient journey, and requiring feasible and valid measures. Stakeholders supported the inclusion of mortality, infection, life participation and kidney function as the core outcomes domains for children with CKD.
\end{abstract}

\title{
Key words:
}

Children

Chronic kidney disease

Dialysis

Transplant

Core outcomes set

Patient-centered care

Outcomes

Trials 


\section{INTRODUCTION}

Children with chronic kidney disease (CKD) have a substantially higher risk of mortality and lifethreatening comorbidities, compared to their age-matched peers ${ }^{1-3}$. Moreover, they have to contend with invasive interventions, appointments, hospitalizations, infections, complex treatment regimens, and lifestyle restrictions ${ }^{4-9}$, whilst also attempting developmental tasks and milestones. Symptoms and side-effects including pain, fatigue, sleep problems, growth restrictions, and changes in their appearance can be highly distressing and debilitating for children with CKD and their families ${ }^{10-13}$. CKD can have a detrimental impact on quality of life (QoL), mental health, and cognitive and psychosocial development ${ }^{1,14-17}$, which can have long-term consequences. Adults diagnosed with CKD during childhood have worse educational, vocational and social outcomes than the general population ${ }^{18-20}$.

Caring for children with CKD is challenging because of the multiple short and longer-term considerations regarding the impact of various treatment options on children with CKD, as well as the competing priorities among children, caregivers and health professionals ${ }^{21}$. Evidence to inform decision-making is limited because the outcomes of critical importance to patients and those involved in their care are either absent or inconsistently measured and reported across trials in children with $\mathrm{CKD}^{22}$. For example, mortality and infection are critically important outcomes for caregivers and children with CKD, yet are only reported in $14 \%$ and $29 \%$ of trials respectively ${ }^{21,22}$.

Children with CKD give high priority to outcomes that threaten their sense of normality - including lifestyle outcomes (for example, ability to attend school or participate in sport), symptoms such as fatigue, and wellbeing $^{21}$. Despite this, less than one per cent of trials in pediatric CKD report QoL, fatigue, depression and school performance ${ }^{22}$. In a systematic review of outcomes reported in pediatric nephrology trials, only 10 $(10 \%)$ of the 100 different outcome domains were patient-reported outcome measures, and these were omitted from the majority of trials ${ }^{22}$. 
The problems with the selection and reporting of outcomes are recognized in nephrology and other medical disciplines, with many initiatives underway to establish core outcomes sets to address this. A core outcomes set is defined as a minimum set of standardized outcomes that are agreed to be measured and reported in all trials in a specified clinical population, because they are critically important to patients and their health professionals $^{23}$. Researchers may add other outcomes that are relevant to the trial aims and intervention. In 2014, the Standardized Outcomes in Nephrology (SONG) initiative was launched to establish core outcomes to improve the relevance and consistency of outcomes reported and measured in trials ${ }^{24}$. Core outcomes sets have been established for hemodialysis, peritoneal dialysis and kidney transplantation in the adult population $^{24}$.

The SONG-Children and Adolescents (SONG-Kids) initiative commenced in 2016 with the aim of developing a core outcomes set to be reported in all trials in children with $\mathrm{CKD}^{9}$. We convened two stakeholder workshops (San Diego, United States, 2018 and Melbourne, Australia, 2019) involving children with CKD, family/caregivers and health professionals, to review and discuss potential core outcomes identified through a standardized consensus process that involved a systematic review ${ }^{22}$, focus groups with nominal group technique ${ }^{21}$, and an international online Delphi survey ${ }^{25}$. This report provides a summary of the discussions at the two international stakeholder workshops and input from the contributors and presents the SONG-Kids core outcomes set.

\section{SONG-KIDS CONSENSUS WORKSHOPS}

\section{Overview and context}

Two SONG-Kids consensus workshops were convened. The first workshop was held on October 27, 2018 in San Diego, United States, during the American Society of Nephrology Kidney Week, and the second workshop was held on April 13, 2019 in Melbourne, Australia, during The International Society of 
Nephrology World Congress of Nephrology. The SONG-Kids consensus workshops were convened to elicit stakeholder feedback on the identification and implementation of a potential core outcomes set for trials in children with CKD. The proposed core outcomes were based on an international two-round Delphi survey that involved children and young people with CKD (aged 8-21 years) $(\mathrm{N}=72)$, caregivers of children with CKD (all ages) $(\mathrm{N}=132)$, and health professionals $(\mathrm{N}=353)$. The survey was conducted in English, French and Hindi languages and included patients and caregivers from 15 countries (the United States, Australia, United Kingdom, Singapore, Canada, India, New Zealand, France, Morocco, Myanmar, Malaysia, Pakistan, Philippines, Portugal and Saudi Arabia). The Delphi survey preceded and informed the workshops.

In round 2, the critically important outcome domains were identified as those with a mean score of $>7$ (on a 9-point Likert scale) across all three stakeholder groups (patients, caregivers, health professionals), median $\geq 8$ across all groups, and where $>75 \%$ in each group scored the outcome 7 to 9 . The most critically important outcome domains across patients, caregivers and health professionals included mortality, life participation, kidney function, infection, cardiovascular disease and blood pressure, which were presented and discussed at the workshops. The detailed analysis and final results of the Delphi survey will be published separately.

\section{Participants and contributors}

We invited children with CKD and young adults with all stages of childhood-onset CKD (aged 7-21 years), caregivers, and health professionals (pediatric nephrologists, adult nephrologists, surgeons, psychiatrists, nursing and allied health professionals, researchers, regulators, policy makers, funders, and industry representatives). We sent targeted invitations to individuals with leadership positions or decision-making roles in professional societies, regulatory agencies (e.g. Food and Drug Administration, Centers for Medicare and Medicaid Services, European Medicines Agency, Australian Pharmaceutical Benefits Scheme/Medical Services Advisory Committee), funding organizations (e.g. National Institutes for Health, 
International Society of Nephrology, the National Health and Medical Research Council of Australia), government and policy organizations (Australian Department of Health), guideline organizations (e.g. Kidney Disease Improving Global Outcomes), registries (e.g. Australian and New Zealand Dialysis and Transplant Registry), and journal editors.

In total, 7 patients, 7 siblings/friends, 24 caregivers, and 43 health professionals from 10 countries attended the first workshop in San Diego (a total of = 81 attendees), and 7 patients, 2 siblings, 23 caregivers and 49 health professionals from 13 countries attended the second workshop in Melbourne (total 81 attendees). The names and affiliations of all attendees and contributors are provided in the acknowledgements.

Patients/caregivers were from the United States, Australia, and Canada. The 92 health professionals were from 18 countries including Australia ( $n=37)$, the United States $(n=23)$, the United Kingdom ( $n=5)$, Canada $(n=4)$, Lithuania $(n=2)$, China $(n=2)$, South Korea $(n=2)$ and one from Belgium, Brazil, France, Germany, India, Italy, New Zealand, Puerto Rico, Singapore, South Africa and Switzerland. The age of patients who participated in the workshops ranged from 7 to 21 years $($ mean $=13$, standard deviation $=4.4)$. The investigators who were unable to attend contributed feedback on the workshop program and the draft workshop report by email. All comments provided in the post-consultation feedback supported the final core outcomes set, therefore no further modifications were made.

\section{Workshop program and process}

The program and materials were circulated to the participants and contributors one week prior to the workshops. During the workshops, we presented the SONG-Kids process and the preliminary results from the SONG-Kids Delphi Survey. At the San Diego workshop, participants were allocated into eight breakout groups, and in Melbourne, participants were allocated to six groups. Each group included a mix of caregivers and health professionals to encourage exchange of diverse perspectives. Children, adolescents, and their siblings/friends) were allocated to a separate group (two groups in San Diego, one group in 
Melbourne) to promote rapport and open discussion. Each group had a trained facilitator who moderated the discussion using a question guide (Supplementary File 1), and at least one member of the SONG-Kids Steering Group or Executive Committee to provide clarification as needed.

Facilitators asked participants to reflect and comment on the potential core outcomes identified in the SONG-Kids Delphi survey. It is recommended that a core outcomes set includes three to five outcome domains for feasibility, and that at least one patient-reported outcome should be included (i.e. an outcome that is measured by the patient's direct report of how they feel and function $)^{26}$. This has been determined by key stakeholders in the nephrology community, including patients, caregivers, health professionals (including trialists) to be feasible and reasonable to implement in trials ${ }^{10-12}$ and follows the approach of core outcomes established for adults receiving kidney replacement therapy ${ }^{4,10,12}$ and other initiatives that are developing core outcomes in other medical specialties ${ }^{4,13}$ The inclusion of a patient-reported outcome is necessary to ensure that the core outcomes set is comprehensive and addresses all broad domains of

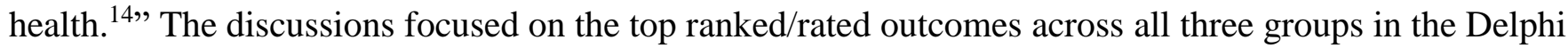
survey (patients, caregivers and health professionals), and these included: mortality, kidney function, life participation, infection, blood pressure and cardiovascular disease. During the final plenary discussions, the Chair (JC) asked the nominated speaker from each breakout group to summarize their discussion.

All breakout and plenary discussions were audiotaped and transcribed verbatim. Transcripts were entered into HyperResearch (ResearchWare Inc, version 3.0) to facilitate coding and analysis of the data. CSH reviewed the transcripts line-by-line, and inductively identified and coded concepts into themes reflecting the range of perspectives on the core outcome domains for trials in pediatric kidney disease. These were discussed among the facilitators and investigators to ensure that it captured the full range and depth of the discussions. 


\section{SUMMARY OF THE DISCUSSIONS}

\section{Overview}

From the discussions across both workshops, we identified four themes relating to establishing a core outcomes set for trials in children with CKD: survival and life participation as common high priority goals; capturing the whole child and family; ensuring broad relevance across the patient journey; and requiring feasible and valid measures. The respective subthemes are described in the following section. Illustrative quotations for each theme are shown in Table 1. The SONG-Kids core outcome domains are shown in Figure 1 (tier 1). Recommendations from the consensus workshops are summarized in Table 2.

$\underline{\text { Survival and life participation are common high priority goals }}$

Wanting to live: For patients, "being alive" was fundamentally important. They feared the possibility of dying from complications, surgical procedures or non-adherence - "No one wants to die... That is why we go into hospital and we put up with all the needles so that we can continue to live our life." Adults, who had experienced CKD in childhood, indicated that death had been at the "forefront of their mind" when they were younger. Caregivers and health professionals were concerned about the child's long-term survival. Some health professionals were surprised that mortality was a high priority for children - "it is interesting to me that kids are thinking about death". Complications and comorbidities, that were viewed as threats to survival (for example, infection and cardiovascular disease) were prioritized by caregivers and health professionals.

Simplifying language around mortality: Patients and caregivers were concerned that the term "mortality" could cause confusion, as some were not familiar with the term. For others, its catastrophic connotations led 
to undue distress. Patients and caregivers suggested to use the term "survival" as this reflected their focus on their child's life expectancy over the longer-term and conveyed a sense of "resilience."

Participating in normal life: Patients wanted to be able to participate in life so they could "be the same as everyone else". Health professionals and caregivers recognized life participation as an overarching goal of care - "improving life participation is the whole point of my job" (health professional) - while some believed it was challenging to address - "we don't see it as something we can treat" (health professional). Participants defined life participation as a "catch-all" domain that broadly included psychological wellbeing, social, academic, vocational and sports participation and independence. Participants suggested that life participation could be impacted by a range of outcomes including hospitalization, disease-related symptoms (e.g. fatigue), mental health, dietary and lifestyle restrictions, the medication regimen and side effects, and complications (e.g. infection).

Catastrophic and debilitating impacts of infection: Infection was seen as a threat to survival (including graft survival) and life participation for children. Infection meant patients had to be hospitalized, and this interfered with family and social functioning, and school attendance - "last year she only attended 55 days out of the entire school year. [It was] urinary tract infections and kidney infections" (caregiver). The fear and constant need to be vigilant to prevent infection explained the high priority given to this outcome. Health professionals agreed that infection was important across all stages of CKD, but noted variability in the severity, prevalence, and recurrence of different types of infections, which would need to be considered in establishing infection as a core outcome domain.

Recognizing the seriousness of mental health: Participants emphasized that mental health and life participation were closely linked. Children and adolescents reported that low-self-esteem and a fear of standing out made them feel less inclined to participate in social activities. Some children felt hopeless and unmotivated to attend school because they were unable to concentrate and could not keep up with their peers 
- "I don’t feel like going to school because why bother? I know I'm going to get tired and breathless." Patients agreed that life participation was more suitable as a core outcome domain than mental health outcomes because it had broader relevance. However, some caregivers and health professionals believed that for specific groups of patients, particularly adolescents, mental health was more important. Some caregivers expressed concerns for their child's "psychological survival" and risk of developing severe mental health problems because of the "trauma" of childhood CKD.

Supporting a patient-centered approach: Patients believed that health professionals should focus on the whole person and address life participation alongside clinical outcomes - "it should be normal to ask a patient 'how do you feel like you are doing at school?"' (patient). Health professionals stated that measuring life participation in trials would enable them to answer questions relevant to patients and caregivers - "if we don't put it in [the trial] we don't even know if kids who take this drug get to go to school or to sleepovers" (health professional).

Capturing the whole child and family

Amplifying the child's voice: Some patients felt unheard in clinical settings and believed their priorities such as life participation (including the ability to participate in school and sports), should be reflected in the core outcomes set. Caregivers agreed that children were often "closed off" and unable to voice their perspectives in clinical consultations, for example regarding emotional issues and life participation. Health professionals also remarked that the involvement of children expanded their views and approach - "it makes me wonder if I am asking enough [e.g. about itching] actually" (health professional).

Relevance of kidney function and blood pressure to daily functioning: Kidney function was viewed as an important indicator of disease status and informative for clinical decision-making - "it dictates everything that you do" (health professional). Health professionals also indicated that blood pressure was a relevant 
marker of the child's health and disease status (e.g. fluid overload, kidney function, transplant function or cyst growth). Some caregivers felt that blood pressure was important because of its association with "day to day" symptoms (e.g. headaches, fatigue, fainting) and because "it's easier to relate to it as a lay person [than cardiovascular disease]". However, some health professionals questioned the inclusion of blood pressure as a core outcome domain, arguing that hypertension is "totally irrelevant" to a large proportion of patients with CKD- "we have a whole lot of kids with CKD with congenital conditions, renal dysplasia, who don’t have hypertension" (health professional).

Protecting the child's future health: Caregivers and health professionals felt responsible to protect the child's future health and thus prioritized outcomes that considered "the duration of the life of the patient". Some health professionals thought that cardiovascular disease should be a core outcome domain because "you have to improve that outcome 30 years earlier...so they are healthier adults". Some patients and health professionals considered that blood pressure may not be as high a priority compared with cardiovascular disease because effective treatments were already available for controlling blood pressure - "I think blood pressure is not affecting us because we are taking tablets daily for the blood pressure. It is more heart health" (patient). Some health professionals viewed blood pressure as a surrogate measure for cardiovascular disease, and believed cardiovascular disease was more critically important - "I'm talking to my patients all the time about blood pressure... because I'm worried about their heart disease in the future" (health professional).

Family impact: Participants recognized the impact of CKD on the wider family, including caregiver burnout, psychological wellbeing, family functioning and financial burdens, and emphasized that the core outcome domains should include outcomes that impact on the family. Infection was considered to cause considerable family disruption, as patients with infection could require hospitalization, and parents would need to take time off work. 
Broad relevance across the patient journey

Attuned to developmental changes: Patients explained how "the things that are important to [them] are changing" with age and emphasized that the core outcome domains should reflect what is important across development. Health professionals believed that this would enable longitudinal assessment of life participation. However, measuring life participation among younger children was expected to be complex. Caregivers also highlighted concerns about the impact of kidney disease on their child's cognitive development and ability to graduate from high school, complete higher education, find employment and maintain relationships. They believed that these outcomes should be captured within the domain of life participation. Health professionals suggested that age-specific outcomes should be excluded from the core outcomes set (e.g. growth may be more relevant to children who developed kidney disease in early-mid childhood rather than late adolescence once growth had finished).

Identifying priorities relevant across the stages of treatment: Life participation, infection, kidney function, survival and blood pressure were considered broadly relevant across all stages of CKD - pre-dialysis, dialysis and post-transplant. While some health professionals acknowledged that trials included specific populations based on treatment stage, a core outcomes set relevant across treatment stages would propel a shift towards a more "systems-based view" of research in pediatric nephrology as children with CKD often transition between stages of treatment. Participants agreed that treatment-specific outcomes (e.g. itching) should be excluded from the core outcomes set, as these could be informed from separate core outcomes sets that were specific for transplantation or dialysis.

$\underline{\text { Requiring feasible and valid measures. }}$

Complexities in measuring long-term clinical outcomes in children: Health professionals suggested that the routine measurement of cardiovascular disease (e.g. using echocardiograms) would not be feasible in the 
context of core outcome measurement. Some health professionals emphasized that blood pressure was easy to measure and more relevant to pediatric kidney disease compared to cardiovascular disease - "we don't see [cardiovascular disease] in the pediatric age range, blood pressure is more important in the short term" (health professional). Participants thought it would not be feasible to "show differences in mortality" in most pediatric trials as "you need vast numbers of patients" and trials in children are typically underpowered for rare events. Health professionals emphasized that there are few if any valid surrogate measures for mortality, cardiovascular disease and end-stage kidney disease in children. The transition of pediatric patients to adult care was also seen as a barrier to long-term outcome measurement.

Needing validated measures for life participation: Participants suggested that life participation seemed to "encompass everything," and the concept may need to be clarified to inform the selection and development of a measure for this outcome. Caregivers and health professionals suggested that the measure may need to include a range of activities: school attendance, playdates, sleepovers and outings with friends/family, holidays or school camps; and emphasized that the measure would need to be relevant across age groups. Patients emphasized the importance of keeping up with their friends in physical activities and being able to play sport. Whilst they suggested the measure should cover activities in a comprehensive way, they preferred a short and simple measure (e.g. school absenteeism).

Capturing idiosyncrasies in life participation: Participants discussed how life participation could be interpreted differently among children with CKD and recommended that a measure of life participation should be relevant to each individual- “one person's good life participation might not be another's”. For example, they suggested a broad question e.g. "can you still do what you could last year?" so participants could respond on their own terms and with reference to their ability to participate in personally relevant activities. Others suggested that the measure must include a range of different domains of participation to ensure broad relevance. Some health professionals also discussed cultural differences in the meaning of life participation, for example in regard to autonomy and independence - "when you talk about a child with 
disease in Asia, it is normal that they stay at home...the parents will be the barrier to the world." (Health professional)".

Avoiding burdens for participants and researchers: Health professionals questioned whether specific outcomes were practical or relevant to measure in all trials. Health professionals suggested that it could be "a burden on families to have that extra endpoint" if researchers are required to measure and report outcomes that are unlikely to be relevant. They considered it important that the measures be feasible to minimize additional burden to patients. However, health professionals argued that without a core outcomes set, we "run the risk of the opposite happening, when it is not reported in trials when you do need to know [the outcome]".

\section{POST-WORKSHOP CONSULTATION}

All participants received a draft workshop report and were asked to provide feedback within a two-week time frame. The SONG-Kids core outcomes set (Figure 1) was sent to all participants for review and comment. We uploaded the core outcomes set on the SONG website for feedback (https://songinitiative.org/projects/song-kids/). Input from the workshop participants and investigators was integrated into the final report.

\section{DISCUSSION}

Patients, caregivers and health professionals identified survival and life participation to be of critical importance for decision-making, emphasizing that these outcomes reflected the goals of care, and therefore should be included in the core outcomes set. Life participation was thought to capture "the whole" child, and incorporated multidimensional aspects of their life including medical, lifestyle, mental health, social functioning, independence and development or 'growing well' in a meaningful way. Patients, caregivers and 
health professionals considered the impact of the outcomes on the family unit. For example, infection requiring hospitalization meant that parents had to take time off work. While blood pressure and cardiovascular disease were considered important, participants recognized that these were not suitable as core outcomes because they were not relevant to all childhood CKD diagnoses and it would not be feasible to mandate measurement of cardiovascular disease in all trials in children with CKD. Key considerations for implementing the core outcome domains included the need to establish a validated pediatric measure for life participation that is applicable throughout childhood, across treatment stages and cultural backgrounds. Health professionals highlighted the need to ensure that the measures would not increase the burden on participants and researchers. Patients and caregivers did not express concerns regarding the burden of outcome measurement.

The discussions from these workshops informed the selection of core outcome domains to be reported in trials in children across the spectrum of CKD (Figure 1, Table 2). The SONG-Kids core outcomes are survival, kidney function, life participation and infection - consistently regarded by all stakeholders to be critically important for decision-making irrespective of the intervention. The term "survival" was used in preference to "mortality" as this was regarded by patients and caregivers to be more meaningful, comprehensible, and less confronting. Cardiovascular disease and blood pressure are in the middle tier (Figure 1). Whilst they are important to stakeholders, the long-term assessment of cardiovascular disease would not be feasible in all trials and blood pressure not relevant to all subgroups among children with CKD. The risk of cardiovascular disease in the adult CKD population is well known, and is captured in core outcomes sets for adults receiving dialysis, or who have received a kidney transplant ${ }^{24}$. Other outcomes that were not identified as critically important core outcomes (e.g. academic performance, impact on family/friends) were included in the second and third tiers of the core outcomes set.

We acknowledge that fewer children attended the workshops compared with parents and health professionals. We recognize that the 14 patients who participated in the workshops were from Australia, the 
United States and Canada, compared with the 72 patients and 132 caregivers from 15 countries who participated in the Delphi Survey, which preceded the workshops. Most participants were from high income countries, and there may be differences in priorities for outcomes in low income countries, in part due to the variability in healthcare systems and access to care. The proposed core outcomes were identified from the international Delphi survey involving 557 participants from 48 countries, with $16 \%$ of patients/caregivers and $23 \%$ of health professionals from low to middle income countries ${ }^{5}$. Some health professionals acknowledged that there were potential differences in the prioritization of outcomes in low income countries, however participants expected that the outcomes mortality, kidney function, infection and life participation would be critically important in low income countries. Some participants noted that there may be differences in the meaning of life participation in different cultures. We suggest that the use of technology could enable a larger number of patients from different countries to participate in consensus workshops. However, the patients who attended the workshop indicated agreement with the top-prioritized outcomes from the Delphi survey.

Several core outcomes sets have been established for other pediatric specialties, though efforts to involve children in the process are sparse. Mortality has also been identified as a core outcome in childhood asthma, epilepsy $^{27,28}$, though not in Type 1 diabetes $^{29}$. All three included quality of life as a core outcome ${ }^{12,27,29}$. To our knowledge, life participation has not been established as a core outcome in the context of childhood chronic disease, however core outcomes recently established for adolescents and young adult patients with spinal deformity included life participation-related domains such as "recreation and leisure."30

Following this workshop, feasible and valid core outcome measures for the core outcome domains will be identified and assessed for use in the pediatric CKD population, or developed where no such measure exists. To establish the core patient-reported outcome of life participation, we will use the Consensus-based Standards for the selection of health Measurement INstruments-Core Outcome Measures in Effectiveness Trials (COSMIN-COMET) framework6. This will involve interviews and consensus workshops with 
children with CKD, families and health professionals to ensure content validity of the measure. Pilot and validation studies will ensure the outcome measures are appropriate, psychometrically robust, and feasible for children with CKD. For life participation, the measure will be simple and short with three to five questions. This process will ensure that the implementation of the outcome measureswill not impose an excessive or undue burden on participants and researchers. This can help to facilitate acceptance and uptake of the core outcomes set. The use of core outcomes in all trials in CKD is likely to improve the consistency of reporting outcomes important to all stakeholders, and thus strengthen the value of trials for informing decisions in practice and policy. 


\section{Acknowledgements}

The authors thank the International Society of Nephrology for supporting the SONG-Kids Consensus workshop at the World Congress of Nephrology on the $13^{\text {th }}$ April, 2019 in Melbourne, Australia. We acknowledge, with permission, all the attendees listed below who attended the consensus workshops.

SONG-Kids Workshop 1 | San Diego, United States: Alicia Neu, Allison Tong, Alyssa Karathanas, Andrea Viecelli, Andrew Narva, Angela Ju, Ankit Sharma, Augustina Jankauskiene, Beth Vogt, Brittney Bscardark, Brooklyn Romeo, Charlotte Logeman, Cheryl Sanchez-Kazi, Christy Perkins, Darien Karathanas, David Hooper, Debbie Gipson, Detlef Bockenhauer, Ed Romeo, Elaine Ku, Elena Levtchenko, Emily Morales, Emmah Carlton, Eric Au, Estefania Morales, Gerilyn Yoder, Germaine Wong, Giselle Morales, Hailey Desmond, Hayley Perkins, Hui-Kim Yap, Il-Soo Ha, Israel Samaniego, Jeff Brockett, Jenny Shen, Jonathan Craig, Joshua Samuels, Kaleb Yoder, Katherine Twombley, Kennedy Romeo, Leslie Lippincott, Lidia Saldiver, Lizett Samaniego, Madelynn Karathanas, Manuel Benavides, Martin Schreiber, Marva Moxey-Mims, Mary Leonard, Matthew Karathanas, Meghan Pearl, Melissa Brockett, Melvin Bonilla, Michael Zappatelli, Mina Matsuda-Abedini, Mini Michael, Mycah Carlton, Nadine Benador, Nick Webb, Nigel Yoder, Paiyton Carlton, Rasheed Gbadegesin, Raymond Benavides, Rebecca Johnson, Ricardo Moarles Jr, Rocio Vargas, Samaya Anumudi, Scott Wenderfer, Shanna Sutton, Skyla Wilson, Susan Lippincott, Susan Mendley, Symone Charles, Talia Gutman, Tiffany Carlton, Timothy Carlton, Virginia Charles, Yeoungjee Cho, Yo Han Ahn, Zachary Perkins.

SONG-Kids Workshop 2 | Melbourne, Australia: Abhjit Guha, Adaobi Solarin, Alexander Hamilton, Alice Woods-Barnard, Allison Tong, Amelia LePage, Andrea Viecelli, Anna Fielding, Anna Francis, Arvind Bagga, Augustina Jankauskiene, Barbara Gillespie, Brenda Hemmelgarn, Camilla Hanson, Chandana Guha, Chanel Prestidge, Charmaine Green, Craig Fielding, Craig Settee, Detlef Bockenhauer, Dieter Haffner, Elisabeth Hodson, Erin Olver, Fatima Mirza, Fiona Mackie, Georges Deschenes, Georgia Plunkett, Germaine Wong, Harrison Mitchell-Smith, Hong Xu, Hui-Kim Yap, Jo Mitchell-Smith, Jo Watson, 
Jonathan Craig, Joshua Kausman, Karine Manera, Karolis Azukaitis, Kelly-Ann McMichael, Kevin Settee, Lachlan McMichael, Lany Trinh, Lorraine Bell, Louese Dunn, Lucinda Barry, Luke Macauley, Madison Mitchell-Smith, Mark Wolfenden, Mignon McCulloch, Mohammad Khan, Ngairre Plunkett, Paige Olver, Raphael Wolfenden, Robert Gardos, Roberto Pescoits-Filho, Rukshana Shroff, Rupesh Raina, Sarah Baldacchino, Sebastian Wolfenden, Sheyma Salih, Simon Carter, Simone Pearson, Simra Khan, Sonia Sharma, Stephen Cornish, Susan Samuel, Talia Gutman, Terri Mitchell-Smith, Yeoungjee Cho, Yusuf Salih, Zeynab Salih 
Figure Legends

Figure 1. SONG-Kids core outcome domains 


\section{Table Legends}

Table 1. Selected quotations from the workshop discussions to illustrate each theme.

Table 2. Workshop recommendations for establishing and implementing core outcome domains for trials in children with CKD 


\section{Supplementary material}

File S1. Facilitator question guide for breakout discussion

Supplementary information is available at Kidney International's website. 


\section{References}

1. Chen K, Didsbury M, van Zwieten A, et al. Neurocognitive and educational outcomes in children and adolescents with CKD: a systematic review and meta-analysis. Clin J Am Soc Nephrol 2018; 13: 387-397.

2. McDonald SP, Craig JC. Long-term survival of children with end-stage renal disease. $N$ Engl J Med 2004; 350: 2654-2662.

3. Mitsnefes MM. Cardiovascular disease in children with chronic kidney disease. J Am Soc Nephrol 2012; 23: 578-585.

4. Dobbels F, Decorte A, Roskams A, et al. Health-related quality of life, treatment adherence, symptom experience and depression in adolescent renal transplant patients. Pediatr Transplant 2010; 14: 216-223.

5. Tjaden L, Tong A, Henning P, et al. Children's experiences of dialysis: a systematic review of qualitative studies. Arch Dis Child 2012; 97: 395-402.

6. Gerson AC, Wentz A, Abraham AG, et al. Health-related quality of life of children with mild to moderate chronic kidney disease. Pediatrics 2010; 125: e349-e357.

7. Diseth $\mathrm{TH}$, Tangeraas T, Reinfjell T, et al. Kidney transplantation in childhood: mental health and quality of life of children and caregivers. Pediatr Nephrol 2011; 26: 1881-1892.

8. McKenna AM, Keating LE, Vigneux A, et al. Quality of life in children with chronic kidney disease_-patient and caregiver assessments. Nephrol Dial Transpl 2006; 21: 1899-1905.

9. Tong A, Samuel S, Zappitelli M, et al. Standardised Outcomes in Nephrology-Children and Adolescents (SONG-Kids): a protocol for establishing a core outcome set for children with chronic kidney disease. Trials 2016; 17: 401.

10. Roumelioti M-E, Wentz A, Schneider MF, et al. Sleep and fatigue symptoms in children and adolescents with CKD: a cross-sectional analysis from the chronic kidney disease in children (CKiD) study. Am J Kidney Dis 2010; 55: 269-280.

11. Tjaden LA, Grootenhuis MA, Noordzij M, et al. Health-related quality of life in patients with pediatric onset of end-stage renal disease: state of the art and recommendations for clinical practice. Pediatr Nephrol 2016; 31: 1579-1591.

12. Wong CJ, Moxey-Mims M, Jerry-Fluker J, et al. CKiD (CKD in children) prospective cohort study: a review of current findings. Am J Kidney Dis 2012; 60: 1002-1011. 
13. Selewski DT, Massengill SF, Troost JP, et al. Gaining the patient reported outcomes measurement information system (PROMIS) perspective in chronic kidney disease: a Midwest pediatric nephrology consortium study. Pediatr Nephrol 2014; 29: 2347-2356.

14. Tjaden LA, Vogelzang J, Jager KJ, et al. Long-term quality of life and social outcome of childhood end-stage renal disease. J Pediatr 2014; 165: 336-342. e331.

15. Mendley SR, Matheson MB, Shinnar S, et al. Duration of chronic kidney disease reduces attention and executive function in pediatric patients. Kidney Int 2015; 87: 800-806.

16. Kogon AJ, Matheson MB, Flynn JT, et al. Depressive symptoms in children with chronic kidney disease. J Pediatr 2016; 168: 164-170. e161.

17. Moreira JM, Soares CMBM, Teixeira AL, et al. Anxiety, depression, resilience and quality of life in children and adolescents with pre-dialysis chronic kidney disease. Pediatr Nephrol 2015; 30: 2153 2162.

18. Tjaden LA, Maurice-Stam H, Grootenhuis MA, et al. Impact of renal replacement therapy in childhood on long-term socioprofessional outcomes: a 30-year follow-up study. Pediatr 2016; 171: 189-195. e182.

19. Bailey PK, Hamilton AJ, Clissold RL, et al. Young adults' perspectives on living with kidney failure: a systematic review and thematic synthesis of qualitative studies. BMJ Open 2018; 8: e019926.

20. Hamilton AJ, Caskey FJ, Casula A, et al. Psychosocial health and lifestyle behaviors in young adults receiving renal replacement therapy compared to the general population: findings from the SPEAK study. Am J Kidney Dis 2019; 73: 194-205.

21. Hanson CS, Gutman T, Craig JC, et al. Identifying Important Outcomes for Young People With CKD and Their Caregivers: A Nominal Group Technique Study. Am J Kidney Dis 2019.

22. Chong LS, Sautenet B, Tong A, et al. Range and heterogeneity of outcomes in randomized trials of pediatric chronic kidney disease. J Pediatr 2017; 186: 110-117. e111.

23. Williamson PR, Altman DG, Bagley H, et al. The COMET handbook: version 1.0. Trials 2017; 18: 280.

24. Tong A, Manns B, Wang AYM, et al. Implementing core outcomes in kidney disease: report of the Standardized Outcomes in Nephrology (SONG) implementation workshop. Kidney Int 2018; 94: 1053-1068.

25. Logeman C GC, Howell M, Hanson CS, Craig JC, Samuel S, Zappitelli M, Matsuda-Abedini M, Dart A, Furth S, Eddy A, Groothoff J, Yap HK, Bockenhauer D, Sinha A, Alexander SI, Goldstein SL, Gipson DS, Michael M, Walker A, Kausman J, Gaillard S, Bacchetta J, Rheault MN, Warady 
BA, Neu A, Christian M, McTaggart S, Liu I, Teo S, Sautenet B, Gutman T, Carter S, Teixeira-Pinto A, Tong A. Developing consensus-based outcome domains for trials in children and adolescents with chronic kidney disease: an international Delphi survey. Am J Kid Dis 2020; Accepted 16th March 2020.

26. SONG Initiative: The SONG Handbook (Version 1.0). In, Sydney, Australia, 2018

27. Sinha IP, Gallagher R, Williamson PR, et al. Development of a core outcome set for clinical trials in childhood asthma: a survey of clinicians, parents, and young people. Trials 2012; 13: 103.

28. Crudgington H, Rogers M, Bray L, et al. Core Health Outcomes in Childhood Epilepsy (CHOICE): Development of a core outcome set using systematic review methods and a Delphi survey consensus. Epilepsia 2019; 60: 857-871.

29. Byrne M, O'Connell A, Egan AM, et al. A core outcomes set for clinical trials of interventions for young adults with type 1 diabetes: an international, multi-perspective Delphi consensus study. Trials 2016; 18: 602 .

30. de Kleuver M, Faraj SSA, Holewijn RM, et al. Defining a core outcome set for adolescent and young adult patients with a spinal deformity. Acta Orthop 2017; 88: 612-618. 
SONG-Kids Consensus Workshops Investigators (attending and non-attending contributors) for group authorship*

\begin{tabular}{|c|c|c|}
\hline Name & Primary Affiliation* & Country \\
\hline Abhjit Guha & SONG Patient/family network & Australia \\
\hline Adaobi Solarin & Lagos State University Teaching Hospital & Nigeria \\
\hline Aditi Sinha & All India Institute of Medical Sciences & India \\
\hline Adriana Platona & Australian Government Department of Health & Australia \\
\hline Alexander Hamilton & University of Bristol & United Kingdom \\
\hline Alice Woods-Barnard & SONG Patient/family network & Australia \\
\hline Alicia Neu & John Hopkins University & United States \\
\hline Allison Tong & The University of Sydney & Australia \\
\hline Allison Dart & University of Manitoba & Canada \\
\hline Allison Eddy & University of British Columbia & Canada \\
\hline Alyssa Karathanas & SONG Patient/family network & United States \\
\hline Amanda Baumgart & The University of Sydney & Australia \\
\hline Amelia Fielding & SONG Patient/family network & Australia \\
\hline Amelia LePage & Monash University & Australia \\
\hline Amelie Bernier-Jean & The University of Sydney & Australia \\
\hline Amy Kelly & The University of Sydney & Australia \\
\hline Ana Teixeira & Hospitalar do Porto & Portugal \\
\hline Andrea Viecelli & University of Queensland & Australia \\
\hline Andrea Matus & The University of Sydney & Australia \\
\hline Andrew Narva & $\mathrm{NIH}$ & United States \\
\hline Angela Ju & The University of Sydney & Australia \\
\hline Angela Yee-Moon Wang & Queen Mary Hospital, The University of Hong Kong & Hong Kong \\
\hline Ankit Sharma & The University of Sydney & Australia \\
\hline Anna Fielding & SONG Patient/family network & Australia \\
\hline Anna Francis & Lady Cilento Children's Hospital & Queensland \\
\hline Anthony Meza & SONG Patient/family network & United States \\
\hline Aria Fielding & SONG Patient/family network & Australia \\
\hline Armando Teixeira-Pinto & The University of Sydney & Australia \\
\hline Arvind Bagga & All India Institute of Medical Sciences & India \\
\hline Augustina Jankauskienè & Vilnius University Institute of Clinical Medicine & Lithuania \\
\hline Ayano Kelly & Australian National University & Australia \\
\hline Barbara Gillespie & University of North Carolina, Covance CRO & United States \\
\hline Benedicte Sautenet & University of Tours & France \\
\hline Beth Vogt & Rainbow Babies and Children's Hospital & United States \\
\hline Bethany Foster & McGill University & Canada \\
\hline Bradley Warady & Children's Mercy Kansas City & United States \\
\hline Bradley Dixon & University of Colorado School of Medicine & United States \\
\hline Braden Manns & University of Calgary & Canada \\
\hline Brenda Hemmelgarn & University of Calgary & Canada \\
\hline Brittney Bscardark & SONG Patient/family network & United States \\
\hline Brooklyn Romeo & SONG Patient/family network & United States \\
\hline Camilla Hanson & University of Sydney & Australia \\
\hline Carlos Meza & SONG Patient/family network & United States \\
\hline Carter Brockett & SONG Patient/family network & United States \\
\hline Chandana Guha & The University of Sydney \& SONG Patient/family network & Australia \\
\hline
\end{tabular}




\begin{tabular}{|c|c|c|}
\hline Chanel Prestidge & Auckland Hospital & New Zealand \\
\hline Charlotte Logeman & The University of Sydney & Australia \\
\hline Charmaine Green & SONG Patient/family network & Australia \\
\hline Cheryl Sanchez-Kazi & Loma Linda University Health & United States \\
\hline Christy Perkins & SONG Patient/family network & United States \\
\hline Claus Peter Schmitt & University Children's Hospital Heidelberg & Germany \\
\hline Craig Fielding & SONG Patient/family network & Australia \\
\hline Craig Settee & Can-SOLVE CKD & Canada \\
\hline Daniel Sumpton & The University of Sydney & Australia \\
\hline Daniel Meza & SONG Patient/family network & United States \\
\hline Darien Karathanas & SONG Patient/family network & United States \\
\hline David Harris & The University of Sydney & Australia \\
\hline David Wheeler & University College London & United Kingdom \\
\hline David Hooper & Cincinnati Children's Hospital & United States \\
\hline Debbie Gipson & University of Michigan & United States \\
\hline Denis Geary & The Hospital for Sick Children & Canada \\
\hline Detlef Bockenhauer & University College London & United Kingdom \\
\hline Dieter Haffner & Hannover Medical School & Germany \\
\hline Djalila Mekahli & KU Leuven-University of Leuven & Belgium \\
\hline Dorota Drozdz & Jagiellonian University Medical college & Poland \\
\hline Ed Romeo & SONG Patient/family network & United States \\
\hline Elaine $\mathrm{Ku}$ & University of California San Francisco & United States \\
\hline Elaine Urbina & Cincinnati Children's Hospital Medical Center & United States \\
\hline Elena Levtchenko & University Hospital Leuven & Belgium \\
\hline Elena Balovlenkov & Centers for Medicaid and Medicare Services & United States \\
\hline Elisabeth Hodson & The University of Sydney & Australia \\
\hline Emily Morales & SONG Patient/family network & United States \\
\hline Emma O'Lone & The University of Sydney & Australia \\
\hline Emma Machuca & SONG Patient/family network & United States \\
\hline Emmah Carlton & SONG Patient/family network & United States \\
\hline Eric $\mathrm{Au}$ & The University of Sydney & Australia \\
\hline Erin Olver & SONG Patient/family network & Australia \\
\hline Estefania Morales & SONG Patient/family network & United States \\
\hline Fatima Mirza & SONG Patient/family network & Australia \\
\hline Fiona Mackie & Sydney Children's Hospital & Australia \\
\hline Francesca Tentori & DaVita & United States \\
\hline Franz Schaefer & University of Heidelberg & Germany \\
\hline Gail Higgins & The University of Sydney & Australia \\
\hline Georges Deschenes & Robert-Debré University Hospital & France \\
\hline Georgia Plunkett & SONG Patient/family network & Australia \\
\hline Gerilyn Yoder & SONG Patient/family network & United States \\
\hline Germaine Wong & Westmead Hospital & Australia \\
\hline Giselle Morales & SONG Patient/family network & United States \\
\hline Greg Germino & National Institutes of Health & United States \\
\hline Hailey Desmond & University of Michigan & United States \\
\hline Hayley Perkins & SONG Patient/family network & United States \\
\hline Harrison Mitchell-Smith & SONG Patient/family network & Australia \\
\hline
\end{tabular}




\begin{tabular}{|c|c|c|}
\hline Helen Coolican & PKD Foundation Australia & Australia \\
\hline Hong $\mathrm{Xu}$ & Children's Hospital of Fudan University & China \\
\hline Hui-Kim Yap & National University of Singapore & Singapore \\
\hline Ifeoma Anochie & University of Port Harcourt Teaching Hospital & Nigeria \\
\hline Il-Soo Ha & Seoul National University Hospital & South Korea \\
\hline Ira Davis & Clinical Trial and Consulting & United States \\
\hline Isaac Liu & National University Hospital & Singapore \\
\hline Israel Samaniego & SONG Patient/family network & United States \\
\hline Jaap Groothoff & Emma Children's Hospital Academic Medical Centre & Netherlands \\
\hline Jaime Machuca & SONG Patient/family network & United States \\
\hline James Machuca & SONG Patient/family network & United States \\
\hline Jasmijn Kerklaan & The University of Sydney & Australia \\
\hline Jeff Brockett & SONG Patient/family network & United States \\
\hline Jenna Norton & National Institutes of Health & United States \\
\hline Jenny Shen & University of California & United States \\
\hline Jens Goebel & University of Colorado School of Medicine & United States \\
\hline Jia Rao & Children's Hospital of Fudan University & China \\
\hline Jimmy Machuca & SONG Patient/family network & United States \\
\hline Jo Mitchell-Smith & SONG Patient/family network & Australia \\
\hline Jo Watson & Australian Government Department of Health & Australia \\
\hline John Gill & University of British Columbia & Canada \\
\hline Jonathan Craig & Flinders University & Australia \\
\hline Joseph T Flynn & University of Washington & United States \\
\hline Joshua Kausman & Royal Children's Hospital Melbourne & Australia \\
\hline Joshua Samuels & University of Texas & United States \\
\hline Justine Bacchetta & University Hospital of Lyon & France \\
\hline Kaleb Yoder & SONG Patient/family network & United States \\
\hline Karine Manera & The University of Sydney & Australia \\
\hline Karolis Azukaitis & Vilnius University, Institute of Clinical Medicine & Lithuania \\
\hline Katherine Twombley & Medical University of South Carolina & United States \\
\hline Kelly-Ann McMichael & SONG Patient/family network & Australia \\
\hline Kenji Ishikura & Tokyo Metropolitan Children's Medical Center & Japan \\
\hline Kennedy Romeo & SONG Patient/family network & United States \\
\hline Kevin Settee & SONG Patient/family network & Canada \\
\hline Kim Linh Van & The University of Sydney & Australia \\
\hline Lachlan McMichael & SONG Patient/family network & Australia \\
\hline Lany Trinh & Australian Institute of Health and Welfare & Australia \\
\hline Larry Greenbaum & Emory University & United States \\
\hline Laura Sanchez & SONG Patient/family network & United States \\
\hline Leo Fielding & SONG Patient/family network & Australia \\
\hline Lesley Rees & Great Ormond Street Hospital for Children & London \\
\hline Leslie Lippincott & SONG Patient/family network & United States \\
\hline Levi Mejia-Saldivar & SONG Patient/family network & United States \\
\hline Lidia Saldivar & SONG Patient/family network & United States \\
\hline Lisa Guay-Woodford & National Institute of Health & United States \\
\hline Lizett Samaniego & SONG Patient/family network & United States \\
\hline Lorraine Hamiwka & University of Calgary & Canada \\
\hline
\end{tabular}




\begin{tabular}{|c|c|c|}
\hline Lorraine Bell & McGill University & Canada \\
\hline Louese Dunn & Sheffield Teaching Hospitals NHS Foundation Trust & United Kingdom \\
\hline Lucinda Barry & Donate Life & Australia \\
\hline Luke Macauley & SONG Patient/family network & Australia \\
\hline Luke Holmes & SONG Patient/family network & Australia \\
\hline Madelynn Karathanas & SONG Patient/family network & United States \\
\hline Madison Mitchell-Smith & SONG Patient/family network & Australia \\
\hline Mandy Walker & Royal Children's Hospital Melbourne & Australia \\
\hline Manuel Benavides & SONG Patient/family network & United States \\
\hline Marcello Tonelli & University of Calgary & Canada \\
\hline Maria Ferris & University of North Carolina Kidney Center at Chapel Hill & United States \\
\hline Marina Vivarelli & Ospedale Pediatrico Bambino Gesù-IRCCS & Italy \\
\hline Mark Wolfenden & SONG Patient/family network & Australia \\
\hline Martin Howell & The University of Sydney & Australia \\
\hline Martin Christian & Nottingham Children's Hospital & United Kingdom \\
\hline Martin Schreiber & DaVita & United States \\
\hline Marva Moxey-Mims & George Washington University & United States \\
\hline Mary Leonard & Stanford University & United States \\
\hline Matthew Karathanas & SONG Patient/family network & United States \\
\hline Meghan Pearl & University of California Los Angeles & United States \\
\hline Melissa Natatmadja & University of Queensland & Australia \\
\hline Melissa Brockett & SONG Patient/family network & United States \\
\hline Melvin Bonilla-Felix & University of Puerto Rico & Puerto Rico \\
\hline Meredith Atkinson & Johns Hopkins University School of Medicine Baltimore & United States \\
\hline Michael Zappitelli & Montreal Children's Hospital & Canada \\
\hline Michel Baum & University of Texas Southwestern Medical Center & United States \\
\hline Michelle Rheault & University of Minnesota & United States \\
\hline Mignon McCulloch & Red Cross War Memorial Children's Hospital & South Africa \\
\hline Mina Matsuda-Abedini & Sick Kids & Canada \\
\hline Mini Michael & Texas Children's Hospital & United States \\
\hline Mohammad Khan & SONG Patient/family network & Australia \\
\hline Mohammad Salih & SONG Patient/family network & Australia \\
\hline Mycah Ann Carlton & SONG Patient/family network & United States \\
\hline Nadine Benador & UC San Diego Health & United States \\
\hline Ngairre Plunkett & SONG Patient/family network & Australia \\
\hline Nick Webb & Novartis & Switzerland \\
\hline Nicki Scholes-Robertson & The University of Sydney & Australia \\
\hline Nicolas Larkins & University of Western Australia & Australia \\
\hline Nicole Evangelidis & The University of Sydney & Australia \\
\hline Nigel Yoder & SONG Patient/family network & United States \\
\hline Norma Meza & SONG Patient/family network & United States \\
\hline Paige Olver & SONG Patient/family network & Australia \\
\hline Paiyton Carlton & SONG Patient/family network & United States \\
\hline Patrick Brophy & University of Iowa & United States \\
\hline Peter Tugwell & University of Ottawa & Canada \\
\hline Pierre Cochat & University Hospital of Lyon & France \\
\hline Rajnish Mehrotra & University of Washington & United States \\
\hline
\end{tabular}




\begin{tabular}{|c|c|c|}
\hline Raphael Wolfenden & SONG Patient/family network & Australia \\
\hline Rasheed Gbadegesin & Duke University Medical Center & United States \\
\hline Raymond Benavides & SONG Patient/family network & United States \\
\hline Rebecca Johnson & $\begin{array}{l}\text { Children's Mercy Hospitals Kansas City/UMKC School of } \\
\text { Medicine }\end{array}$ & United States \\
\hline Ricardo Morales Jr & SONG Patient/family network & United States \\
\hline Richard McGee & Children's Hospital at Westmead & Australia \\
\hline Richard Fish & University Hospitals of North Midlands & United Kingdom \\
\hline Robert Gardos & PKD Australia & Australia \\
\hline Roberto Pecoits-Filho & Pontifícia Universidade Católica do Paraná & Brazil \\
\hline Rocio Vargas & SONG Patient/family network & United States \\
\hline Rodolfo Saldivar & SONG Patient/family network & United States \\
\hline Rosanna Coppo & Regina Margherita University Children's Hospital & Italy \\
\hline Rukshana Shroff & University College London & United Kingdom \\
\hline Rupesh Raina & Akron Children's Hospitals, Akron Ohio & United States \\
\hline Sajeda Youssouf & Barts Health NHS Trust & United Kingdom \\
\hline Sally Crowe & Crowe Associates Ltd & United Kingdom \\
\hline Samaya Anumudu & Baylor College of Medicine & United States \\
\hline Samuel Chan & Princess Alexandra Hospital & Australia \\
\hline Sarah Baldacchino & Kidney Health Australia & Australia \\
\hline Scott Wenderfer & Baylor College of Medicine & United States \\
\hline Sebastian Wolfenden & SONG Patient/family network & Australia \\
\hline Selah Carlton & SONG Patient/family network & United States \\
\hline Shanna Sutton & SONG Patient/family network & United States \\
\hline Shannon Murphy & University of North Carolina Kidney Center at Chapel Hill & United States \\
\hline Sharon Teo & National University Hospital & Singapore \\
\hline Sheyma Salih & SONG Patient/family network & Australia \\
\hline Shobha Sahney & Loma Linda University Health & United States \\
\hline Silas Carlton & SONG Patient/family network & United States \\
\hline Simon Carter & University of Sydney & Australia \\
\hline Simone Pearson & SONG Patient/family network & Australia \\
\hline Simra Khan & SONG Patient/family network & Australia \\
\hline Skyla Wilson & SONG Patient/family network & United States \\
\hline Sonia Sharma & Max Superspeciality Hospital, New Delhi & India \\
\hline Stephen Alexander & The Children's Hospital at Westmead & Australia \\
\hline Stephen Marks & Great Ormond Street Hospital for Children & United Kingdom \\
\hline Stephen Cornish & Kidney Health Australia & Australia \\
\hline Stuart Goldstein & Cincinnati Children's Hospital Medical Center & United States \\
\hline Susan Samuel & University of Calgary & Canada \\
\hline Susan Furth & University of Pennsylvania & United States \\
\hline Susan Mendley & National Institutes of Health & United States \\
\hline Susan Lippincott & SONG Patient/family network & United States \\
\hline Symone Charles & SONG Patient/family network & United States \\
\hline Talia Gutman & University of Sydney & Australia \\
\hline Terri Mitchell-Smith & SONG Patient/family network & Australia \\
\hline Tess Harris & PKD International & United Kingdom \\
\hline Thorsten Vetter & European Medicines Agency & United Kingdom \\
\hline Tiffany Carlton & SONG Patient/family network & United States \\
\hline
\end{tabular}




\begin{tabular}{lll}
\hline Timothy Carlton & SONG Patient/family network & United States \\
\hline Uwe Querfeld & Charite & Germany \\
\hline Valeria Saglimbene & University of Sydney & Australia \\
\hline Virginia Charles & SONG Patient/family network & United States \\
\hline Wim van Biesen & University of Ghent & Belgium \\
\hline Wolfgang Winkelmayer & Baylor College of Medicine & United States \\
\hline Yenissey Machuca & SONG Patient/family network & United States \\
\hline Yeoungjee Cho & University of Queensland & Australia \\
\hline Yusuf Salih & SONG Patient/family network & Australia \\
\hline Yo Han Anh & Seoul National University Hospital & South Korea \\
\hline Zachary Perkins & SONG Patient/family network & United States \\
\hline Zeynab Salih & SONG Patient/family network & Australia \\
\hline
\end{tabular}

*SONG Patient/family network is indicated for patients, family members and caregivers 


\title{
Table 1. Selected quotations from workshop discussions
}

\author{
Theme/subtheme Illustrative quotation
}

Survival and life participation are common high priority goals

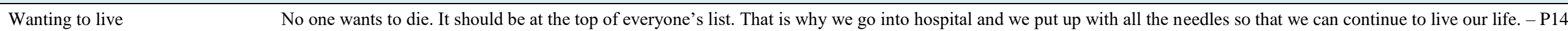
As a child, I was always concerned with 'oh my god, what if I die, who is going to take care of my sister?' Now that I'm older it is more realistic things for me to worry about... So dying isn't at the forefront of my mind... I have accepted that we are all going to die anyway. - C/P1

I just want her to live a long and happy life. I know she doesn't look after herself properly. I can only do so much. - C13

I was quite surprised that mortality was ranked number 2 for kids. I have rarely heard any of my patients saying that they are afraid to die. Or that they even realize they can die. I know that they might think about it but they aren't voicing it very often. - H3

Simplifying language around mortality
Survival is also a healthy mind. It's not just survival of the organs and body functioning. We were worried about [our daughter's] psychological survival" - C11.

You want long term survival for your child. It is sort of a flip side; it is more of a positive slant than a negative slant - H11

We talked about the rephrasing of mortality, to survival. With more positive connotations. And more holistic from physical to psychological- Plenary, Melbourne.

I have family members with kidney disease. [Mortality] is not in our daily conversation. We are always thinking about life and enjoying the moment. Physicians are always focused on mortality but that is so far away... we think about how much time they have left and how we can enjoy it. - C/H11

We don't have the technical background, simplified language helps on a patient/caregiver side. - C10

Participating in a normal life

That should be our core thing. I can't run like other kids. I can't do what they do. I'm always behind and you are basically different you are out of the group like you are a differen person. $-\mathrm{P} 14$

We can't do stuff like other people. Such as work and sports activities. - P14

In life participation there is a side for us and there is a side for the medical side. What can the medical side do to help us have a better life participation? Then also help us understand where that line is, because obviously it's never going to be normal for them. - C5

We have done things like go on vacation when our doctor did not necessarily want us to and I feel like for us it was so good for our son...the doctor is medical and looking at mortality and for us you look at trying to live life the way we want to. $-\mathrm{C} 2$

We got to make sure that they can survive on the drug, and that they can live a normal life on the drug. - C5

Life participation may be things like going to school, playing with friends, go to sleep overs, go to birthday parties, being able to eat. - H2

Catastrophic and debilitating impacts of infection
Before I was diagnosed, I was very sporty and I had lots of friends and then when I got sick I avoided them because I knew I wasn't allowed to get sick. And when I'd get sick, I'd get really sick... a whole bunch of people are sick around me. It's flu season". - P14

Swimming is a big 'no, no'. Dancing. Footy is the big thing at the moment. She wants to be just like every other twelve-year-old. Her infection rates are off the chart. Last year, she only attended 55 days out of the entire school year. Urinary tract infections and kidney infections. - C9

The word infection has been part of her life since the time she was born. We have had infection pre transplant during renal failure and after renal failure. I'm fearful that she is going to have an infection and we are going to end up in hospital again. For us the word infection is something we live with. - C13

[Infection] affects many aspects. Some don't eat very well. It affects their growth. Especially the babies. It's a vicious cycle. - H9

To me life participation is huge for my children because we can all go through the infection pathway and knock ourselves out of participation. - C9

If you look at the core outcomes in layer 2 the infection impacts on almost all the outcomes in that layer too. Anxiety and stress, cognition, depression, fatigue, growth, ability to travel, almost all of them are impacted by recurring infection so I think that is pretty important. - C10

As pediatric nephrologists CAKUT is one of the major causes of kidney disease and infection is one of the major things that we deal with. - H11

"In a country like India, there is a really high rate of infection." - H12 
I do have terrible self-esteem and it's gotten slightly worse because now I have to worry about all these medications I have to take and they are really time sensitive... for example we are going to the beach, 'time to leave because he needs meds'. - P7

I worry about my friends, being bullied - P8

Self-esteem is important because it would affect the way they participate too. He doesn't smile with his teeth. He has low self-esteem. That is from medicine. And that is why we are very interested in what his medicine does to him in the long term. $-\mathrm{C} 10$

The emotional and psychological, we are suffering through what I would call PTSD from being in hospital. The pokes the prods, all the procedures. Of all the procedures...that we have to battle through now. So that is why I say we should maybe not be so quick to dismiss their views... - C2

The mental health aspect seems to be a really big thing in the adolescents that we look after and the adults you receive from us. So much anxiety. - H12 It does impact on their participation in life. Depressed patients withdraw from the school.... $-\mathrm{H} 12$

Supporting a patient-centered approach
It should be normal to ask a patient, 'how do you feel you are doing at school?' Not the main premise

It is important because it translates those mortality and kidney function numbers into real life. It generalizes what is happening. This person had that treatment and this person was able to do whatever to improve their life so I think it is pretty important for that reason. $-\mathrm{C} 13$

It is sometimes hard to get at self-esteem exactly in a quick clinic visit. How you are feeling about yourself? It doesn't really come up in that way. Well if they come in and are like I learnt these great things in school yesterday we kind of get a sense that they are doing well and participating. So, I always ask about life participation. I feel like as providers that is how we are measuring that. $-\mathrm{H} 3$

\section{Capturing the whole child and family}

Amplifying the child's voice

I've been seeing my renal doctor since I was little and she doesn't want to believe that I'm changing as a person and that things that are important to me are changing. When I was younger I would have said, itching, bone health, self-esteem would have been a big issue, but now that I'm older, I don't really care about it as much but she still insists that my treatment has to revolve around that and mum agrees with her and they don't listen to me. It should be based around the patients' ideas because we're the ones dealing with it. - P7 To throw out the opinions of others just because they are younger is not fair with what the patients inevitably have to endure. - Plenary, San Diego

Relevance of kidney function and blood pressure to daily functioning

$\mathrm{BP}$ for us is vital because she goes very symptomatic with blood pressure issues. - C 9

BP is really up there. You can tell so much from those little outcomes. CVD I haven't personally focused on, looked into or understood much about. - C9

Hypertension is going to make the kidney disease worse and we need to preserve what kidney function she's got and keep on top of it. - C10

We tend to pay less attention to long term cardiovascular outcomes as pediatric nephrologists. On a day to day basis what we battle is BP. $-\mathrm{H} 10$

Kidney function certainly is important as a marker of where you are in terms of kidney disease towards dialysis and transplant and whether you need to start worrying about it or what you need to implement. I would have thought that was pretty key at every phase. It dictates everything that you do. - H13

We have a whole lot of kids with kidney disease, with congenital conditions, renal dysplasia, who don't have hypertension at the beginning. - H9

For children, the key reasons why we worry about BP is a) that it can affect the kidney function and b) it can affect cardiovascular health. Therefore, I feel it is not an independent outcome measure. $-\mathrm{H} 10$

Protecting the child's future

health BP isn't affecting us because we are taking tablets daily for the BP. I'm thinking heart health is more important. - P14

I can deal with if he is the littlest kid. What's most important is what's absolutely the best, the best outcome for his transplant kidney to survive the longest. - C 5

I saw blood pressure and I thought huh, that's because I'm talking to my patients all the time about blood pressure, blood pressure, blood pressure. But actually it's because I'm worried about their heart disease in the future. Trying to protect their hearts. - H3

Providers think about kidney function, cardiovascular disease and mortality because we know that those are the things that are going to impact the duration of life of the patient. For a lot of the younger children it is a more immediate thing. $-\mathrm{H} 1$

Family impact

Other infections, also gastroenteritis, it escalates. It means coming to hospital when you don't need to. Spending money coming to hospital. For everyone, the patient, family, extended family. $-\mathrm{H} 9$

Since it happened to my daughter at only six months old, so I guess more of a journey will be more for my wife and I. $-\mathrm{Cl}$

If he goes to hospital, I miss work, and I'm thinking missing work, being away from my other child so that's huge. - C5

It impacts on her sister. She gets jostled around because of doctor's appointments, or if we have to run her to the hospital because she has a fever. - C6 


\section{Broad relevance across the patient journey}

Attuned to developmental

changes
If you don't keep growing, it will be a very bad thing, if your mind doesn't keep growing, you can't really do much - P8

More of the social, how successful are they, how many are getting accepted into college, how many are graduating high school, those successes in life - C6

More research should be going into social outcomes, life outcomes, productivity in the world and in society, my son is 16, he struggles with life in general. - C4

What is most important for my son is that he develops cognitively. Before he was born those were the types of questions, I would ask our nephrologist. How is he going to develop cognitively as opposed to his peers going forward? And of these, what captures that? Is it life participation? - C2

When you are talking about children moving through adolescence. Some of those things become more critical in terms of their anxiety, stress, depression. - H10

Identifying priorities relevant across the stages of treatment Terms like life participation probably mean the same to all three groups but some of the other terms have different weighting depending on which of the three categories you belong to. $-\mathrm{H} 13$

A systems-based view of the journey. How do we smooth those rough spots out? And acknowledge that it's the same patient going through different parts of the journey and it's not a transplant population, a dialysis population, its patients going through different phases. $-\mathrm{H} 1$

I wonder if there were a lot of patients on hemodialysis. With some exceptions, they are almost always the only ones complaining about itching. - H3

\section{Requiring feasible and valid measures}

Complexities in measuring longterm clinical outcomes in children

$\mathrm{BP}$ is measured at every consultation and as they get older, they might start doing it at home and bringing it in, in a diary. It is of direct relevance to our clinical practice. Other measures like ECHOs or cardiovascular mortality we don't do those all the time. - H12

What is CVD? If you ask me that? It's not easy in the young child to assess in a standardized way. If I do a study in small children, let's go for the hypertension first because this we can monitor and it is the best surrogate. If it is older children, we can do other things. - H1

Blood pressure might mean something else in the pediatric population. Especially in the setting of glomerular diseases. It might not really be long-term cardiovascular disease. It might be short term active disease. - H1

Needing validated measures for

They often talk about number of days they miss at school or work. They don't go out with friends they don't stay overnight at parties. They don't want to go out with a group of friends without their mother or father because they are anxious. They might need to go home quickly. It's trying to get a sense of what is a comparison. It's not fool proof. - H10 A lot of it is subjective and we can't ask a two-year-old. The number of days missed is objective. - H10

You could ask someone how much school they have missed because this is a combination of social development, cognitive development all these things. - C2 Self-esteem, life participation and impact on family and friends. I automatically think about how that would be evaluated and measured. - H3

Capturing idiosyncrasies in life participation

You should ask are you still able to do what you could do person up and do something at the earlier stage. - H12

I don't know how we are going to measure it and it is going to be very subjective. One person's good life participation might not be another's. - H13

Asian parents are really protective of their children. They keep the children at home and that is normal for the family in Asia. In this culture [Western culture] this will be bad, but in a conservative Asian family it is normal. So therefore, it is very hard to standardize the impact of the disease [on life participation] and use the same measure. - H1

Avoiding burdens for participants and researchers

That [mortality] would be very hard to capture in a pediatric study I would think because it would happen some years later. - H10

Should it be included in a trial that scientifically is not going to have an impact on infections. It is a burden on families to have that extra endpoint. - H13

But if you don't have a core outcome are we going to run the risk of the opposite happening when it is not reported in trials when you do need to know it. - H13

$\mathrm{H}$, health professional, $\mathrm{P}$, patient, $\mathrm{S}$, sibling, $\mathrm{C}$, caregivers, number indicated is group ID (1-8 San Diego, 9-14 Melbourne) 
Table 2. Summary of workshops recommendations to consider in establishing core outcome domains for trials in children with CKD

\section{Core outcome domains for trials in children with CKD should:}

- Capture fundamental goals of life and care that are of high priority to patients, caregivers and health professionals (i.e. life participation, survival)

- $\quad$ Consider the child as a whole

- Consider the child's development and life course through to adulthood

- Consider the impact on the family, particularly as children depend on their parents for care

- Reflect outcomes to be addressed in patient-centered care and shared decision-making because they are critically important regardless of whether they may be responsive to interventions

- Be communicated using terms that are comprehensible and meaningful (i.e. use the term survival instead of mortality)

- Include clinical outcomes that may be life-threatening, disruptive and debilitating (e.g. infection)

- Include outcomes that supports clinical decisions, self-management and are relevant indicators of health (i.e. kidney function)

- Be relevant for patients with diverse demographic and clinical characteristics (e.g. age, culture, diagnosis)

- Be applicable for children irrespective of stage of treatment

- Be feasible to implement in all trials

Implementation of core outcomes requires

- $\quad$ Outcome measures that are feasible, simple and validated in children with CKD

- Measures that are easy to administer/collect with minimal burden on patients, caregivers and researchers 